\title{
Anthocyanins protect human endothelial cells from mild hyperoxia damage through modulation of Nrf2 pathway
}

\author{
Francesco Cimino - Antonio Speciale - Sirajudheen Anwar • \\ Raffaella Canali • Elisabetta Ricciardi · Fabio Virgili • \\ Domenico Trombetta $\cdot$ Antonina Saija
}

Received: 11 September 2012/Accepted: 18 October 2012/Published online: 16 November 2012

(C) Springer-Verlag Berlin Heidelberg 2012

\begin{abstract}
The detrimental effects of high oxygen supplementation have been widely reported. Conversely, few is known about the effects of exposure to mild hyperoxic conditions, an interesting issue since the use of oxygenenriched mixture is now increasingly used in clinical practice and especially for professional and recreational reasons. Our study investigated if in vitro exposure of human umbilical vein endothelial cells (HUVECs) to moderate hyperoxia $\left(\mathrm{O}_{2} 32 \%\right)$ induces cellular alterations, measured as changes in cell signaling pathways. Furthermore, by means of an ex vivo experimental model where human volunteers were used as bioreactors, we studied whether anthocyanin metabolites are able to protect HUVECs against mild hyperoxia-induced damage. We observed that the cytotoxic effect of mild hyperoxia came along with a significant decrease in nuclear accumulation of the transcription factor Nrf2, as well as in the expression of Nrf2-regulated antioxidant and cytoprotective genes. Furthermore, under normoxic conditions, anthocyanin metabolites appeared able to activate the Nrf2 pathway, through the involvement of specific kinases (ERK1/2); this adaptive effect may explain the protective effect observed in mild hyperoxia-exposed HUVECs following anthocyanin pretreatment. This study confirms that dietary anthocyanins
\end{abstract}

Cimino F. and Speciale A. contributed equally to this work.

F. Cimino - A. Speciale $\cdot$ S. Anwar · E. Ricciardi .

D. Trombetta $(\square) \cdot$ A. Saija

Department Farmaco-Biologico, University of Messina,

Viale Annunziata, 98168 Messina, Italy

e-mail: dtrombetta@unime.it

R. Canali · F. Virgili

National Research Institute for Food and Nutrition,

Via Ardeatina 546, 00178 Rome, Italy and/or their metabolites can protect endothelial cells against mild hyperoxia-induced alterations acting as cell signaling modulators.

Keywords Mild hyperoxia - Anthocyanins - Endothelial cells $\cdot$ Cellular adaptive response $\cdot$ Nrf2/ARE pathway

$\begin{array}{ll}\text { Abbreviations } \\ \text { ARE } & \text { Antioxidant responsive element } \\ \text { AS } & \text { Anthocyanin serum } \\ \text { ERK } & \text { Extracellular signal-regulated kinases } \\ \text { FRAP } & \text { Ferric reducing/antioxidant power } \\ \text { FS } & \text { Fasting serum } \\ \text { GAPDH } & \text { Glyceraldehyde-3-phosphate dehydrogenase } \\ \text { HO-1 } & \text { Heme oxygenase-1 } \\ \text { Keap1 } & \text { Kelch-like ECH-associated protein 1 } \\ \text { MAPKs } & \text { Mitogen-activated protein kinases } \\ \text { MAPKK } & \text { Mitogen-activated protein kinase kinase } \\ \text { NQO-1 } & \text { NAD(P)H:quinone oxidoreductase 1 } \\ \text { Nrf2 } & \text { NF-E2-related factor-2 } \\ \text { ROS } & \text { Reactive oxygen species } \\ \text { TEAC } & \text { Trolox equivalent antioxidant capacity }\end{array}$

\section{Introduction}

High oxygen concentrations (hyperoxia) can cause cell injury and death, through not-completely understood mechanisms associated with reactive oxygen species (ROS) formation and inflammatory responses (Gore et al. 2010). In fact, an overly produced ROS is detrimental because it significantly damages cell structures such as lipids, proteins and nucleic acids resulting in relevant alteration of health status (Wells et al. 2009). On the other 
hand, slightly increased ROS may be beneficial because ROS may also act as secondary messengers in the intracellular signal transduction as well as in a variety of cell processes in different biological systems (Dröge 2002; Thom 2009) and in particular those related to the physiological handling of oxygen bioavailability (Genestra 2007).

The detrimental effects of ventilation with pure oxygen or very high oxygen concentration have been well reported (Altemeier and Sinclair 2007). Although respiratory diseases causing hypoxemia, such as pneumonia and neonatal surfactant deficiency, often necessitate the use of supplemental oxygen as part of their management, it is known that administering high oxygen concentrations for prolonged periods may contribute to cause lung injury (such as acute respiratory distress syndrome in adults and bronchopulmonary dysplasia in preterm infants), targeting especially the pulmonary vasculature and producing endothelial injury and eventually loss of vascular integrity (Pagano and Barazzone-Argiroffo 2003; Phillips and Tsan 1988)

Conversely, few is known about the effects of exposure to mild hyperoxic conditions. There is a large interest in this issue since the use of oxygen-enriched mixture, such as EAN (Enriched Air Nitrox), containing $32 \% \mathrm{O}_{2}$ and also $\mathrm{N}_{2}$, is now increasingly used in clinical practice and especially for professional and recreational reasons (i.e., scuba diving).

The main aim of our study was to investigate the cellular alterations induced by in vitro exposure of human umbilical vein endothelial cells (HUVECs) to moderate hyperoxia $\left(\mathrm{O}_{2} 32 \%\right)$, measured as changes in cell signaling pathways, in comparison with normoxia conditions $\left(\mathrm{O}_{2}\right.$ $21 \%)$. Furthermore, we wanted to demonstrate that, through dietary supplementation, anthocyanins or their metabolites are able to protect HUVECs against mild hyperoxia-induced damage. In fact, several scientific studies suggest that some polyphenols contained in Mediterranean plant food and generally referred to as antioxidants may inhibit the oxidative damage and restore endothelial function in order to slowdown the development of atherogenic injury. Among these compounds, anthocyanins are known for their effectiveness, including cyanidin3-O-glucoside $(\mathrm{C} 3 \mathrm{G})$ that is probably the most studied among the others. However, phenolic phytochemicals may exert their effects independently from the antioxidant capacity, interacting at different levels with cellular signaling pathways (Speciale et al. 2011b). In our experiments, we employed an experimental model mimicking the complex human metabolic system, in which cells were fed with human serum taken after supplementation with the anthocyanins mixture and then containing only absorbed compounds and/or metabolites (Canali et al. 2010).

\section{Materials and methods}

Reagents

Methanol, ethanol, acetonitrile, chloroform and isopropyl alcohol were obtained from CarloErba Reagent (Milan, Italy) in their highest commercially available purity grade. All other reagents, if not specified, were purchased from Sigma-Aldrich (Milan, Italy).

Dietary supplementation for ex vivo experiments

To obtain the serum to be used in the following ex vivo experiments, a dietary anthocyanin supplement was given to 4 healthy men volunteers (aged 35-40 years). The subjects were asked to consume, in fasting conditions, a unique dose (net wt $390 \mathrm{mg}$ ) of Medox ${ }^{\circledR}$ (Biolink Group AS, Sandnes, Norway), a dietary supplement whose $41 \%$ $(160 \mathrm{mg})$ is represented by purified anthocyanins isolated from bilberries (Vaccinium myrtillus) and black currant (Ribes nigrum). The total anthocyanin content consisted of 17 different natural purified anthocyanins (Qin et al. 2009). The amount of anthocyanins was determined based on a previous human study (Qin et al. 2009).

To determine changes in serum antioxidant activity, blood samples were collected immediately before (fasting serum, FS) and $2 \mathrm{~h}$ after $\operatorname{Medox}^{\circledR}$ administration (AS, anthocyanin serum). In fact, this time was chosen because preliminary experiments showed that the maximum change in serum antioxidant activity appears $2 \mathrm{~h}$ following Medox ${ }^{\circledR}$ administration (data not shown).

Serum was isolated by centrifugation at $1,800 \times g$ for 10 min. Both AS and FS serums were then used to feed human umbilical vein endothelial cells. This study has been conducted in full accordance with the Declaration of Helsinki, and a written informed consent was obtained from each volunteer.

Human serum Trolox equivalent antioxidant capacity (TEAC)

Trolox equivalent antioxidant capacity (TEAC) in human serum was determined by decoloration of the radical cation of 2,2-azinobis-(3-ethylbenzothiazoline-6-sulfonic acid) $\left(\mathrm{ABTS}^{+}\right)$, in terms of absorbance quenching at $740 \mathrm{~nm}$. Briefly, this method determines the capacity of antioxidants to quench the $\mathrm{ABTS}^{+}$radical (Tomaino et al. 2010). The antioxidants inhibit the reaction leading to an absorbance decrease, and the extent of inhibition is proportional to the antioxidant concentration in the sample. Values obtained for each sample were compared with the concentrationresponse curve of a standard Trolox solution, and expressed as mmol of Trolox equivalent antioxidant capacity per 
$\mathrm{L}$ of serum (TEAC mmoles/L serum). Each analysis was carried out in triplicate.

Human serum ferric reducing/antioxidant power (FRAP)

The principle of this method is based on the reduction of a ferric-tripyridyltriazine complex to its colored ferrous form in the presence of antioxidants (Morabito et al. 2010). Briefly, the FRAP reagent contained $2.5 \mathrm{~mL}$ of a $10 \mathrm{mM}$ TPTZ (2,4,6-tripyridyl-s-triazine, Fluka) solution in $40 \mathrm{mM} \mathrm{HCl}, 2.5 \mathrm{~mL}$ of $20 \mathrm{mM} \mathrm{FeCl}_{3}$ and $25 \mathrm{~mL}$ of $0.3 \mathrm{M}$ acetate buffer, $\mathrm{pH}$ 3.6. It was prepared freshly and warmed at $37{ }^{\circ} \mathrm{C}$. The absorbance of the reaction mixture at $593 \mathrm{~nm}$ was measured spectrophotometrically after incubation at $37{ }^{\circ} \mathrm{C}$ for $10 \mathrm{~min}$. $\mathrm{FeSO}_{4} 1 \mathrm{mM}$ was used as standard solution. The final result was expressed as the concentration of antioxidants having a ferric reducing ability equivalent to that of $1 \mathrm{mM} \mathrm{FeSO}_{4}$.

Cell culture and hyperoxic conditions

Human umbilical vein endothelial cells (HUVECs) were isolated from freshly obtained human umbilical cords by collagenase digestion of the interior of the umbilical vein as described elsewhere (Speciale et al. 2011a) and were cultured in medium 199, supplemented with $20 \%$ of fetal bovine serum (FBS), $1 \%$ L-glutamine, $20 \mathrm{mM}$ hepes, 100 units $/ \mathrm{mL}$ penicillin/streptomycin, $50 \mathrm{mg} / \mathrm{ml}$ endothelial cell growth factor and $10 \mu \mathrm{g} / \mathrm{mL}$ of heparin, in gelatinpretreated flasks. Cells were maintained at $37{ }^{\circ} \mathrm{C}$ in an incubator with a humidified atmosphere containing $5 \%$ $\mathrm{CO}_{2}$. Cells used in this study were from the second to fourth passage.

Afterward cells were incubated in hyperoxic $\left(32 \% \mathrm{O}_{2}\right)$ or normoxic $\left(21 \% \mathrm{O}_{2}\right)$ conditions at $37{ }^{\circ} \mathrm{C}$. Mild hyperoxia was produced using a modular incubator gas chamber (M.I.C.101 modular-incubator, Billups-Rothenberg Co.). The chamber was purged with $32 \% \mathrm{O}_{2}$ for 4 min at a flow rate of $20 \mathrm{~L} / \mathrm{min}$ and re-flushed after $1 \mathrm{~h}$ according BillupsRothenberg Co. protocol. All the reagents used to manage cells treated in hyperoxic conditions were also flushed with $32 \% \mathrm{O}_{2}$.

In a preliminary series of experiments, HUVECs were incubated under mild hyperoxic conditions for different times $(0,16,24,48 \mathrm{~h})$. On the basis of cell viability under these experimental conditions, we have choosen $24 \mathrm{~h}$ time exposure for all the other experiments.

\section{Pharmacological treatments}

To investigate the possible protective effect of polyphenolic compounds/metabolites contained in the AS,
HUVECs were washed with DPBS and pretreated for $24 \mathrm{~h}$ with medium supplemented with $20 \%$ AS or FS.

In some experiments carried out to investigate the role of two protein kinases belonging to the mitogen-activated protein kinases (MAPK) family, ERK1 and ERK2 (extracellular signal-regulated kinases), in the observed results, the selective pharmacological inhibitor of mitogen-activated protein kinase kinase (MAPKK), PD98059, was used. PD98059, dissolved in DMSO, was added to cell culture media, at a final concentration of $25 \mu \mathrm{M}, 1 \mathrm{~h}$ before the addition of AS or FS.

\section{Cytotoxicity assay}

The cytotoxic effect of hyperoxia was evaluated by the trypan blue dye exclusion test. Briefly, $10 \mu \mathrm{l}$ of cell suspension was mixed with $30 \mu$ trypan blue isotonic solution $(0.4 \% \mathrm{w} / \mathrm{v})$ and loaded into a hemocytometer for both live and dead cell counting. To avoid interferences, the same test was also used to evaluate human serum cytotoxicity in HUVECs.

\section{Quantitative RT-PCR}

Total cellular RNA was isolated according to the TRIzol protocol. The quality of the RNA was tested in $1 \%$ formaldehyde-agarose gel stained with ethidium bromide (EtBr), and spectrophotometrically quantified. After reverse transcription (RT) with oligo $(\mathrm{dT})_{15}$ primers, polymerase chain reaction (PCR) was performed for identification of HO-1 and NQO-1 mRNA levels. Glyceraldehyde-3-phosphate dehydrogenase (GAPDH) was used as housekeeping gene for normalization.

Gene expression was assessed by real-time PCR (Applied Biosystem 7300 Real-Time PCR System, Monza, Italy) coupled with the Sybr green JumpStart ${ }^{\mathrm{TM}}$ Taq Ready Mix kit. The specific primers set for the target genes are as follows: GAPDH, foward, 5'-GGC TCT CCA GAA CAT CAT CCC TGC- $3^{\prime}$, reverse, $5^{\prime}$-GGG TGT CGC TGT TGA AGT CAG AGG-3' (Canali et al. 2007; Speciale et al. 2011); HO-1, forward, 5'-CAACATCCAGCTCTTTGAGG-3', reverse, 5'-AGAAAGCTGAGTGTAAGGAC-3'; NQO-1, forward, 5'-AAGAGCACTGATCGTACTGG-3', reverse, 5'-CTTC AGTTTACCTGTGATGTCC- $3^{\prime}$. Cycling conditions were 40 cycles of $94{ }^{\circ} \mathrm{C}$ denaturation $(15 \mathrm{~s}), 60{ }^{\circ} \mathrm{C}$ annealing and extension $(1 \mathrm{~min})$. A final dissociation stage was run to generate a melting curve for verification of amplification product specificity. Each sample was assayed at least three times from the same RNA. Data were collected and processed with SDS 1.3.1 software (Applied Biosystems, Monza, Italy) and given as threshold cycle $(\mathrm{Ct})$. The fold increase compared with the control cells not treated and not exposed to hyperoxia mRNA expression was determined using the $2^{-\Delta \Delta \mathrm{Ct}}$ method 
(Livak and Schmittgen 2001). Primer efficiencies for the test genes were comparable to those for GAPDH (reference gene).

Nuclear and cytosolic lysate preparation

Following appropriate treatment, nuclear and cytosolic lysates were prepared as follows. Briefly, cells were lysed for $10 \mathrm{~min}$ at $2{ }^{\circ} \mathrm{C}$ in a hypotonic buffer $(10 \mathrm{mM}$ Hepes, $1.5 \mathrm{mM} \mathrm{MgCl}_{2}, 10 \mathrm{mM} \mathrm{KCl}$ and $5 \%$ glycerol, $\mathrm{pH} 7.8$ ), containing a cocktail of protease inhibitors $(2 \mu \mathrm{g} / \mathrm{ml}$ aprotinin, $1 \mu \mathrm{g} / \mathrm{ml}$ leupeptin and $1 \mathrm{mM}$ benzamidine) and $1 \mathrm{mM}$ DTT, and treated with $0.65 \%$ Igepal (Sigma) for $5 \mathrm{~min}$. Nuclei were recovered by centrifugation at $20,000 \mathrm{~g}$ for 1 min at $4{ }^{\circ} \mathrm{C}$, and the supernatant was kept as the cytoplasmic extract. Nuclear proteins were obtained by incubating with a hypertonic buffer $(20 \mathrm{mM}$ Hepes, $400 \mathrm{mM}$ $\mathrm{NaCl}, 1 \mathrm{mM} \mathrm{MgCl}_{2}, 0.1 \mathrm{mM}$ EDTA, $1 \mathrm{mM}$ EGTA, $10 \%$ glycerol, $\mathrm{pH}$ 7.8) also containing a cocktail of protease inhibitors $(2 \mu \mathrm{g} / \mathrm{ml}$ aprotinine, $1 \mu \mathrm{g} / \mathrm{ml}$ leupeptine and $1 \mathrm{mM}$ benzamidine) and $1 \mathrm{mM}$ DTT. All the protein fractions were stored at $-70{ }^{\circ} \mathrm{C}$ until use. Protein concentration in lysates was determined using Bradford reagent.

\section{Western blotting analysis}

For immunoblot analyses, $40 \mu \mathrm{g}$ of protein lysates per sample were denatured in $4 \times$ SDS-PAGE sample buffer (Tris-HCl 260 mM, pH 8.0, $40 \%$ (v/v) glycerol, $9.2 \%$ (w/v) SDS, $0.04 \%$ bromophenol blue and 2-mercaptoethanol as reducing agent) and subjected to SDS-PAGE on $10 \%$ acrilamide/bisacrilamide gels.

Separated proteins were transferred to nitrocellulose membrane (Hybond-P PVDF, Amersham Bioscience). Residual binding sites on the membrane were blocked by incubation in TBST (10 mM Tris, $100 \mathrm{mM} \mathrm{NaCl}, 0.1 \%$ Tween 20) with $5 \%(\mathrm{w} / \mathrm{v})$ nonfat milk powder overnight at $4{ }^{\circ} \mathrm{C}$. Membranes were then probed with specific primary antibodies: rabbit anti-Nrf2 polyclonal antibody (Santa Cruz Biotechnology) (1:200); rabbit anti-p-ERK1/2 polyclonal antibody (Santa Cruz Biotechnology) (1:150); mouse anti-Lamin B monoclonal antibody (Santa Cruz Biotechnology) (1:200); rabbit anti-cytoskeletal actin (Bethyl Laboratories) (1:5,000), followed by peroxidaseconjugated secondary antibody HRP-labeled goat antirabbit Ig (BD Pharmigen) (1:5,000) and visualized with an ECL plus detection system (Amersham Biosciences). The equivalent loading of proteins in each well was confirmed by Ponceau staining and actin or lamin B control.

Statistical analysis

All the experiments were performed in triplicate and repeated three times. Results are expressed as mean \pm SD from three experiments and statistically analyzed by a one-way ANOVA test, followed by Tukey's HSD, using the statistical software ezANOVA (http://www.sph.sc.edu/comd/ rorden/ezanova/home.html). Differences in groups and treatments were considered significant for $P<0.05$.

\section{Results}

Human serum antioxidant activity

In order to evaluate the changes in serum antioxidant power following anthocyanins-enriched mixture intake, serum antioxidant activity was analyzed by means of TEAC and FRAP tests. Higher levels of both TEAC and FRAP were detected in serum obtained from blood samples withdrawn $2 \mathrm{~h}$ after Medox ${ }^{\circledR}$ intake (AS), when compared with serum from blood collected immediately before Medox ${ }^{\circledR}$ administration (FS) (Table 1).

Time-dependent effect of $32 \% \mathrm{O}_{2}$ on HUVECs viability

As specific information addressing the sensitivity of HUVECs to mild hyperoxic conditions is not available in the literature, we sought to assess the cytotoxic effects of mild hyperoxia $\left(32 \% \mathrm{O}_{2}\right)$ on this primary cell line. At this aim, HUVECs were exposed to hyperoxic stress for 16, 24 and $48 \mathrm{~h}$. Mild hyperoxia induced a time-related cytotoxic effect (as evaluated by the trypan blue dye exclusion test), with a significant decrease in cell viability at $16 \mathrm{~h}$ from the beginning of hyperoxia and steadily increasing at 24 and $48 \mathrm{~h}$. The 24-h exposure time point, inducing a mild cell damage (30\% of cell death), was, therefore, chosen for following experiments (Fig. 1).

\section{Effects on mild hyperoxia-induced cytotoxicity}

The cytotoxic effects of mild hyperoxia was evaluated, by means of the trypan blue dye exclusion test, on HUVECs pretreated for $24 \mathrm{~h}$ with medium containing $20 \%$ AS or FS and then exposed for $24 \mathrm{~h}$ to $32 \% \mathrm{O}_{2}$ (mild hyperoxic conditions) or $21 \% \mathrm{O}_{2}$ (normoxic conditions). The pretreatment with both AS or FS did not affected cell viability of HUVECs exposed to normoxic conditions (Fig. 2). On the contrary, only AS pretreatment was able to significantly protect cells from death induced by mild hyperoxia (Fig. 2).

Activation of the Nrf2/ARE pathway

In order to study the potential mechanisms underlying the damage induced in HUVECs by mild hyperoxia and the protection exerted by polyphenol compounds/metabolites 
Table 1 Serum TEAC and FRAP before and after administration of a unique dose $(390 \mathrm{mg})$ of Medox ${ }^{\circledR}$, containing $160 \mathrm{mg}$ of purified anthocyanins

\begin{tabular}{lll}
\hline & TEAC $(\mathrm{mmoles} / \mathrm{L}$ serum $)$ & $\mathrm{FRAP}^{(\mu \mathrm{M} \text { Fe }}{ }^{2+} / \mathrm{L}$ serum $)$ \\
\hline Fasting serum & $2.88 \pm 0.05$ & $629.5 \pm 23.2$ \\
Anthocyanin serum & $3.34 \pm 0.02^{*}$ & $706.1 \pm 25.1^{*}$ \\
\hline
\end{tabular}

Results are expressed as mean \pm SD from three independent experiments

$A S$ human serum obtained after Medox ${ }^{\circledR}$ capsules consumption, FS human serum obtained immediately before Medox ${ }^{\circledR}$ capsules consumption $* P<0.05$ versus FS

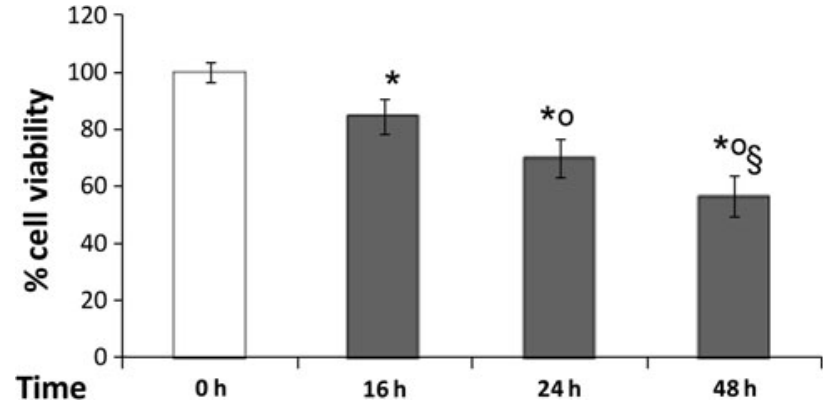

Fig. 1 HUVECs viability after 16-, 24- or 48-h exposure to $32 \% \mathrm{O}_{2}$, as evaluated by the trypan blue assay. The results were compared with those obtained at time $0 \mathrm{~h}$ (control). Results are expressed as mean $\pm \mathrm{SD}$ of at least three experiments. Bars represent the percentage of viable treated cells (mean percentage) versus control. $* P<0.05$ versus $0 \mathrm{~h} .{ }^{\circ} P<0.05$ versus cells exposed to mild hyperoxic conditions for $16 \mathrm{~h} .{ }^{\S} P<0.05$ versus cells exposed to mild hyperoxic conditions for $24 \mathrm{~h}$

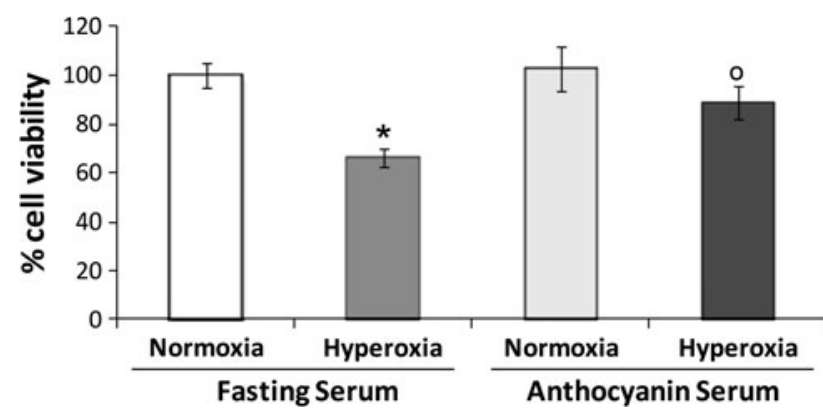

Fig. 2 Effects of 24-h pretreatment with AS or FS on viability of HUVECs exposed for $24 \mathrm{~h}$ to $32 \% \mathrm{O}_{2}$ (mild hyperoxic conditions) or to $21 \% \mathrm{O}_{2}$ (normoxic conditions), as evaluated by the trypan blue assay. Cells treated with FS and exposed to normoxic conditions were used as negative controls. Results are expressed as mean $\pm \mathrm{SD}$ of three experiments. Data represent percentage of viable cells (mean percentage) calculated from the number of viable cells in treated samples versus negative control. $* P<0.05$ versus FS-treated cells exposed to normoxic conditions. ${ }^{\circ} P<0.05$ versus FS-treated cells exposed to hyperoxic conditions. AS, human serum obtained after Medox ${ }^{\circledR}$ capsules consumption; FS, human serum obtained immediately before Medox ${ }^{\circledR}$ capsules consumption

in these toxic conditions, we evaluated under these experimental settings the level of Nrf2 (NF-E2-related factor-2) nuclear localization and the gene expression of two well-known antioxidant enzymes containing ARE sequences within the promoter, namely heme oxygenase-1 (HO-1) and NAD(P)H:quinone oxidoreductase 1 (NQO-1).

Mild hyperoxia induced a significant decrease in the nuclear levels of the transcription factor Nrf2. Interestingly, pretreatment for $24 \mathrm{~h}$ with AS significantly increased Nrf2 nuclear levels in HUVECs subsequently exposed to normoxic conditions (Fig. 3). The alteration induced by $32 \% \mathrm{O}_{2}$ was partially prevented by cells pretreatment with AS; in fact, the increase in Nrf2 nuclear localization induced by AS treatment still remains evident in cells later exposed for $24 \mathrm{~h}$ to mild hyperoxia (Fig. 3).

A similar trend was observed as to the expression of both HO-1 and NQO-1 (Fig. 4). In fact, higher intracellular mRNA levels of the two genes were measured in AS-pretreated cells under normoxic conditions, in comparison with those treated with FS (Fig. 4). Similarly to that found for Nrf2 nuclear localization (Fig. 3), HUVECs exposure to mild hyperoxia induced a significant decrease in the expression of the both HO-1 and NQO-1; this effect was partially but significantly prevented by cells pretreatment with AS but not with FS (Fig. 4).

These findings are in agreement with literature data indicating that dietary polyphenols can induce the expression of genes encoding for antioxidant and detoxifying proteins through the activation of the Nrf2/ARE pathway (Speciale et al. 2010).

\section{Effects on MAPKs phosphorylation}

In basal conditions, Nrf2 translocation is inhibited by the cytoplasmic repressor Kelch-like ECH-associated protein 1 (Keap1). Phase II enzyme inducers and pro-oxidants have been reported to cause modification directly on Nrf2Keap1 complex resulting in Nrf2 release from Keap1, or indirectly causing ERK 1/2 (extracellular signal-regulated kinases 1/2) phosphorylation that in turn activates Nrf2 and its subsequent translocation into the nucleus.

Thus, in order to investigate the upstream mechanism by which hyperoxic conditions and/or polyphenol compounds/ metabolites can modulate Nrf2/ARE pathway in HUVECs, the activation of two protein kinases belonging to the mitogen-activated protein kinases (MAPK) family, ERK1 


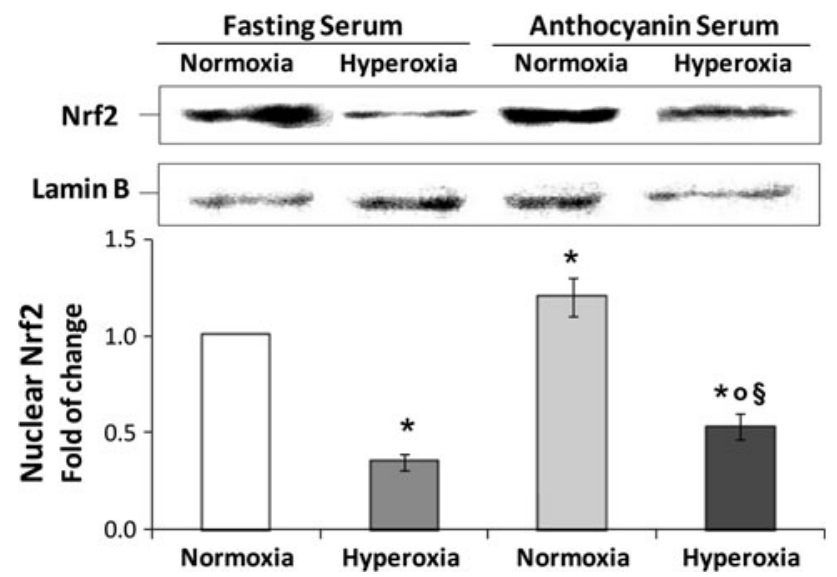

Fig. 3 Effects of 24-h pretreatment with AS or FS on Nrf2 nuclear localization in HUVECs exposed for $24 \mathrm{~h}$ to $32 \% \mathrm{O}_{2}$ (mild hyperoxic conditions) or to $21 \% \mathrm{O}_{2}$ (normoxic conditions). The figure shows a representative image from three independent experiments. Nrf2 bands intensity values were normalized to the corresponding Lamin B values. Results by densitometry are reported as fold change against FS in normoxia and expressed as mean \pm SD of at least three independent experiments. ${ }^{*} P<0.05$ versus FS-treated cells exposed to normoxic conditions. ${ }^{\circ} P<0.05$ versus FS-treated cells exposed to hyperoxic conditions. ${ }^{\S} P<0.05$ versus AS-treated cells exposed to normoxic conditions. AS, human serum obtained after Medox ${ }^{\circledR}$ capsules consumption; FS, human serum obtained immediately before Medox ${ }^{\circledR}$ capsules consumption

and ERK2, was studied. These two protein kinases, usually localized into the cytoplasm in an inactive form under physiological conditions, are considered as the main effectors for Nrf2 nuclear translocation. Cytoplasmic levels of p-ERK1/2 were assessed by means of an antibody recognizing Thr 202 phosphorylated ERK 1 and Thr 184 phosphorylated ERK 2.

We found ERK1/2 phosphorylation (i.e., activation) was induced by AS in cells under normoxic conditions, thus supporting a direct involvement of MAPK cascade in Nrf2/ ARE pathway activation. Furthermore, ERK1/2 phosphorylation appeared to be inhibited by mild hyperoxia (Fig. 5); as seen above for the induction of Nrf2 nuclear localization (Fig. 3) and for the expression of genes containing ARE sequences (Fig. 4), this effect was partially prevented by pretreatment with AS.

To further investigate the relevance of Nrf2 activation pathway on the effects of AS in HUVECs, the MAPKK pharmacological inhibitor PD98059 was used at a concentration $(25 \mu \mathrm{M})$ not cytotoxic by itself under normoxic conditions (data not shown). PD98059 induced a further inhibition of Nrf2 nuclear translocation (without increasing cytotoxicity) in hyperoxia-exposed HUVECs; furthermore, PD98059 heavily hampered AS-induced protective effects against hyperoxia-induced cell death (Fig. 6) and Nrf2 nuclear localization (Fig. 7).
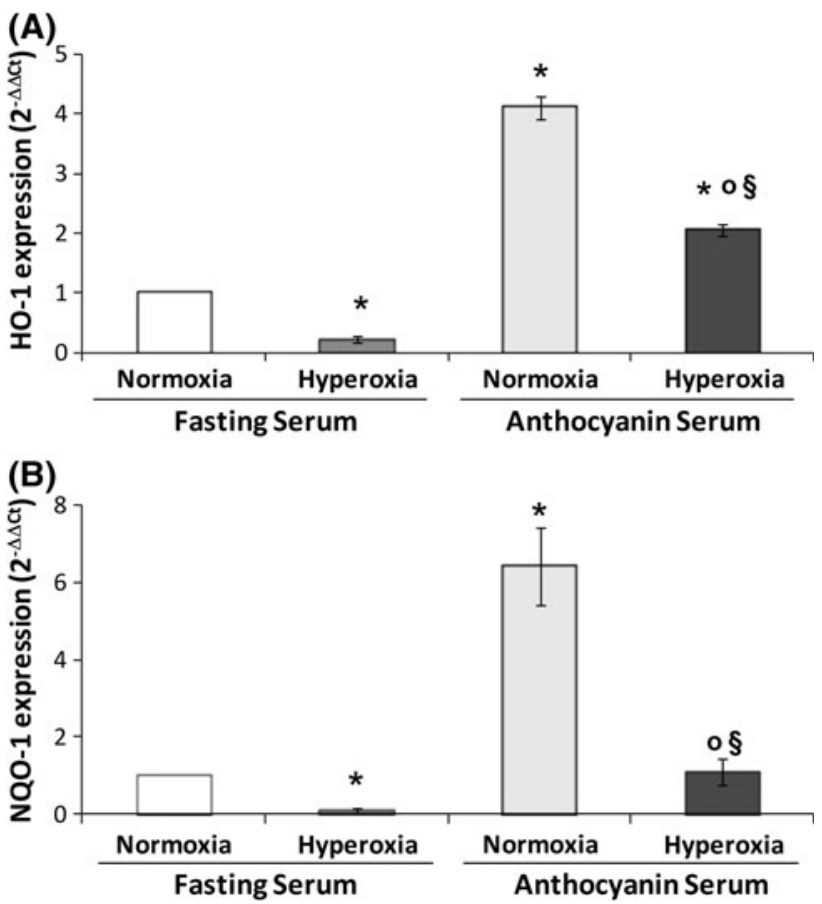

Fig. 4 Effect of 24-h pretreatment with AS or FS on HO-1 and NQO1 gene expression in HUVECs exposed for $24 \mathrm{~h}$ to $32 \% \mathrm{O}_{2}$ (mild hyperoxic conditions) or to $21 \% \mathrm{O}_{2}$ (normoxic conditions). Cells treated with FS and exposed to normoxic conditions were used as negative control. Values are expressed as $2^{-\Delta \Delta \mathrm{Ct}}$ normalized to FStreated cells exposed to normoxic conditions. $* P<0.05$ versus FS-treated cells exposed to normoxic conditions. ${ }^{\circ} P<0.05$ versus FS-treated cells exposed to mild hyperoxic conditions. ${ }^{\S} P<0.05$ versus AS-treated cells exposed to normoxic conditions. a HO-1, heme oxygenase-1; b NQO-1, NAD(P)H:quinone oxidoreductase 1. AS, human serum obtained after Medox ${ }^{\circledR}$ capsules consumption; FS, human serum obtained immediately before Medox ${ }^{\circledR}$ capsules consumption

These results clearly indicate that the activation of the Nrf2-ARE pathway and the modulation of the ERK1/2 phosphorylation is one of the main mechanisms by which polyphenolic compounds/metabolites can exert their beneficial effects against hyperoxia-induced endothelial toxicity, also supporting the essential role of cell adaptive responses elicited by these compounds in their capability to protect cells against stressor agents.

\section{Discussion}

Redox cellular homeostasis is a complex phenomenon crucial for cellular well-being. A large number of experimental results support the involvement of free radicals and oxidative stress in development and progression of numerous diseases, while an enhanced antioxidant status is associated with reduced risk of several diseases. In fact, ROS cause damage to cellular proteins, lipids and nucleic acids, etc., often leading to cumulative organ injury (Wells 


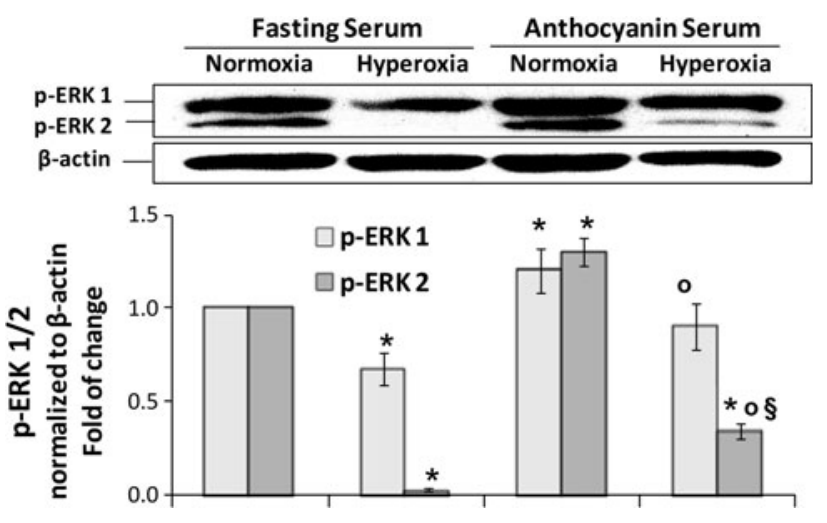

Fig. 5 Effect of 24-h pretreatment with AS or FS on ERK1 and ERK2 phosphorylation in HUVECs exposed for $24 \mathrm{~h}$ to $32 \% \mathrm{O}_{2}$ (mild hyperoxic conditions) or to $21 \% \mathrm{O}_{2}$ (normoxic conditions). Cells treated with FS and exposed to normoxic conditions were used as negative controls. The figure shows a representative image from three independent experiments. Results by densitometry are reported as fold change against control and expressed as mean \pm SD of three experiments. p-ERK1/2 bands intensity values were normalized to the corresponding $\beta$-actin value. $* P<0.05$ versus FS-treated cells exposed to normoxic conditions. ${ }^{\circ} P<0.05$ versus FS-treated cells exposed to mild hyperoxic conditions. ${ }^{\S} P<0.05$ versus AS-treated cells exposed to normoxic conditions. AS, human serum obtained after Medox ${ }^{\circledR}$ capsules consumption; FS, human serum obtained immediately before Medox ${ }^{\circledR}$ capsules consumption

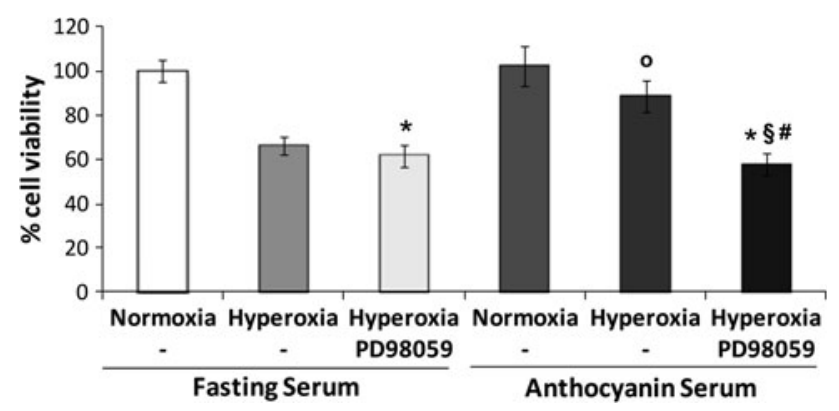

Fig. 6 Effect of MAPKK inhibition on HUVEC viability pretreated for $24 \mathrm{~h}$ with AS or FS and then exposed for $24 \mathrm{~h}$ to $32 \% \mathrm{O}_{2}$ (mild hyperoxic conditions) or to $21 \% \mathrm{O}_{2}$ (normoxic conditions), as evaluated by the trypan blue assay. Cells were incubated with the pharmacological MAPKK inhibitor PD98059 $(25 \mu \mathrm{M})$ for $1 \mathrm{~h}$ before AS or FS addition. Cultures treated with FS and exposed to normoxic conditions were used as negative controls. Each point represents mean \pm SD of three experiments. Data represent percentage of viable cells (mean percentage) calculated from the number of viable cells in each sample versus negative control. ${ }^{*} P<0.05$ versus FS-treated cells exposed to normoxic conditions. ${ }^{\circ} P<0.05$ versus $F S$-treated cells exposed to mild hyperoxic conditions. ${ }^{\S} P<0.05$ versus AS-treated cells exposed to normoxic conditions. ${ }^{\#} P<0.05$ versus AS-treated cells exposed to mild hyperoxic conditions. AS, human serum obtained after Medox ${ }^{\circledR}$ capsules consumption; FS, human serum obtained immediately before Medox ${ }^{\circledR}$ capsules consumption

et al. 2009). In order to protect against oxidative stress, organisms have developed antioxidant systems consisting of thiol containing small molecules, such as glutathione and thioredoxin or enzymes like catalase, glutathione peroxidase and peroxiredoxins (Oktyabrsky and Smirnova 2007). Various studies demonstrated the importance of the activation of Nrf2-regulated transcriptional response in the protection against hyperoxia, a condition which can result in transient oxidative stress. Nrf2 binds to the consensus sequence called antioxidant responsive element commonly found in the regulatory regions (promoter and/or enhancers) of genes encoding several cytoprotective enzymes and proteins and up-regulates their transcription in response to a variety of stimuli (Speciale et al. 2011b). In unstressed cells, Nrf2 is predominantly localized in the cytoplasm in association with Keap1, which promotes proteasomal degradation of Nrf2. Oxidant or toxic insults may perturb the tertiary structure of Keap1, thereby impeding Nrf2 degradation and facilitating Nrf2 nuclear accumulation and subsequent ARE-mediated transcription (Speciale et al. 2011b). The role of the activation of Nrf2-regulated transcriptional response in the protection against hyperoxia $\left(>95 \%\right.$ of $\mathrm{O}_{2}$ )-induced injury was investigated in mice lacking Nrf2 expression and activity (Cho et al. 2002; Reddy et al. 2009). In these studies, 95-99\% of $\mathrm{O}_{2}$ exposure enhanced expression of Nrf2 mRNA and functionally activated nuclear Nrf2 in the lungs of normal $(w t)$ mice, contributing to the protection against hyperoxic lung injury, while a significant attenuation in basal and/or hyperoxia-induced mRNA expression of NQO-1 and HO-1 was observed in the lungs of mice deficient in Nrf2, suggesting that these enzyme genes are downstream effector molecules transcriptionally activated by Nrf2 in the lungs of mice. Furthermore, exposure of human lung microvascular endothelial cells to hyperoxia $\left(95 \% \mathrm{O}_{2}\right)$ stimulates Nrf2 translocation from the cytoplasm to the nucleus and increases Nox4 expression (Pendyala et al. 2011). These results suggest that the use of Nrf2 activators may represent a new therapeutic strategy to prevent and attenuate hyperoxia insult through up-regulation of Nrf2 signaling.

However, all studies reported in literature on this subject have been conducted in experimental conditions using pure oxygen or high concentrations of oxygen ( $>95 \%$ ), but few is known about the effects of exposure to mild hyperoxia, and on the cellular signaling pathways which may be critical in the development of such effects. For this reason, the goal of our study was, instead, to investigate the alterations induced in HUVECs by exposure to mild hyperoxia $\left(\mathrm{O}_{2} 32 \%\right)$. Interestingly, the cytotoxic effect following this experimental condition (Fig. 1) came along with a significant decrease in the nuclear levels of the transcription factor Nrf2 (Fig. 3), as well as with a consequent decrease in the expression of both the investigated ARE-genes, HO-1 and NQO-1 (Fig. 4). Thus, this appears to be a good model to investigate if protection against hyperoxia endothelial damage may be elicited by Nrf2 activators, including selected dietary plant phenolics 


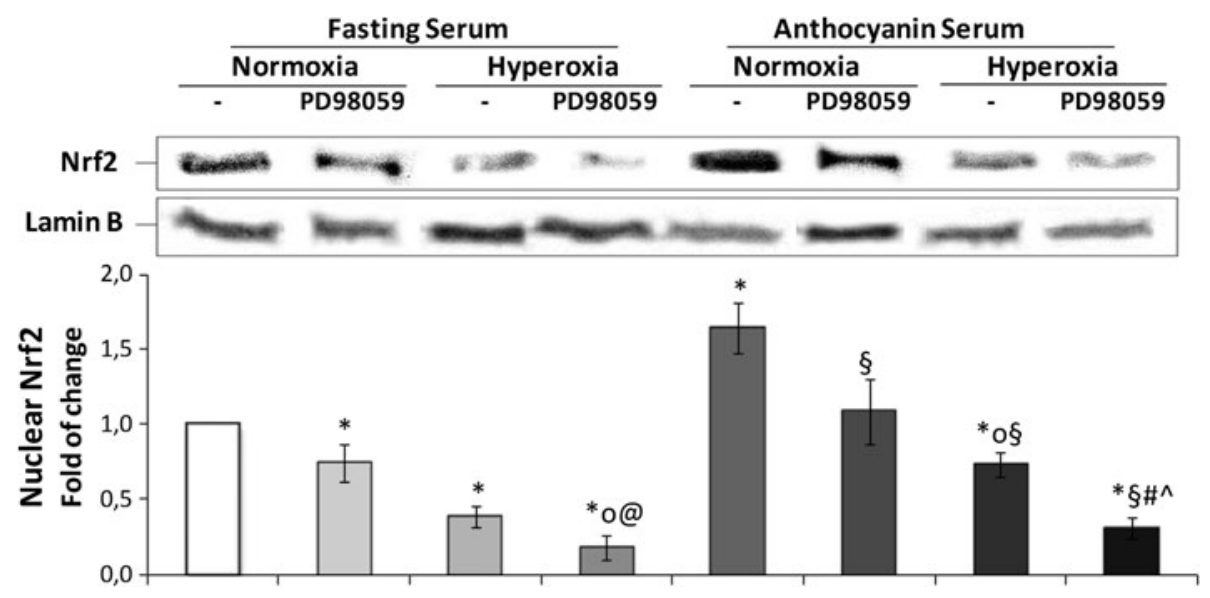

Fig. 7 Effect of MAPKK inhibition on the Nrf2 activation pathway in HUVECs pretreated for $24 \mathrm{~h}$ with AS or FS and then exposed for $24 \mathrm{~h}$ to $32 \% \mathrm{O}_{2}$ (mild hyperoxic conditions) or to $21 \% \mathrm{O}_{2}$ (normoxic conditions), as evaluated by Western blot analysis. Cells were incubated with the pharmacological MAPKK inhibitor PD98059 $(25 \mu \mathrm{M})$ for $1 \mathrm{~h}$ before AS or FS addition. Cultures treated with FS and exposed to normoxic conditions were used as negative controls. Each point represents mean $\pm \mathrm{SD}$ of three experiments. The figure shows a representative image from three independent experiments. Results by densitometry are reported as fold change against controls and expressed as mean $\pm \mathrm{SD}$ of three experiments. Nrf2 bands intensity

known to have beneficial health effects not only for antioxidant activity but also as modulators of the Nrf2 system (Rahman et al. 2006; Speciale et al. 2011a). However, many currently used in vitro experimental models are able to identify, at cellular and molecular levels, responses induced by phytocomplexes or individual phytochemicals but are usually conducted by adding them directly to the culture medium. In our study, we used a new ex vivo experimental protocol, representing an improvement to current in vitro cellular models, with a more physiological approach (Canali et al. 2010). In fact, taking into account that healthy subjects may be used as "bioreactors," we fed endothelial cells with the human serum (AS) taken after supplementation with the anthocyanins mixture and then containing only absorbed compounds and/or metabolites. In normoxic conditions, HUVEC treatment with AS induces a significant increase in Nrf2 nuclear accumulation activation and in HO-1 and NQO-1 expression (Figs. 3, 4), when compared with cells fed with FS. On the other hand, pretreatment for $24 \mathrm{~h}$ with AS partially prevented the decrease in Nrf2 nuclear accumulation and in HO-1 and NQO-1 gene expression, observed in HUVECs subsequently exposed to mild hyperoxia (Figs. 3, 4). These findings confirm that the intake of Medox ${ }^{\circledR}$ is able to produce serum levels of anthocyanin compounds/metabolites high enough to perform a pharmacological effect, as observed also by antioxidant assays (Table 1). Furthermore, the observed protective effect of AS against mild hyperoxia-induced endothelial damage can be ascribed to the activation of the values were normalized to the corresponding Lamin B values. ${ }^{*} P<0.05$ versus FS-treated cells exposed to normoxic conditions. ${ }^{\circ} P<0.05$ versus FS-treated cells exposed to hyperoxic conditions. ${ }^{\circledR} P<0.05$ versus FS-treated cells exposed to hyperoxic conditions and treated with PD98059. ${ }^{\S} P<0.05$ versus AS-treated cells exposed to normoxic conditions. ${ }^{\#} P<0.05$ versus AS-treated cells exposed to hyperoxic conditions. ${ }^{\wedge} P<0.05$ versus AS-treated cells exposed to hyperoxic conditions and treated with PD98059. AS, human serum obtained after Medox ${ }^{\circledR}$ capsules consumption; FS, human serum obtained immediately before Medox ${ }^{\circledR}$ capsules consumption

Nrf2/ARE pathway. Our findings are in agreement with those reported by several papers which report that plant phenols can protect endothelial cell against different stressor conditions by pharmacological activation of the Nrf2/ ARE pathway (Speciale et al. 2011b; Surh et al. 2008).

Nrf2 nuclear translocation requires the activation of several signal transduction pathways as well as MAPKs (Shen et al. 2004). MAPKs (ERKs, JNKs and p38) are downstream effectors in antioxidant responses, and their activities are manifested in activation of many transcription factors, including Nrf2 (Limón-Pacheco et al. 2007). Previous studies have shown that some MAPKs, like ERK1/2, play a role in the induction of Nrf2 (Filomeni et al. 2012; Speciale et al. 2011a). However, the exact role of MAPKs in Nrf2 activation, as well as the underlying molecular mechanism, remains poorly understood. In our experimental conditions, manipulation of the catalytic activity of ERK1 and ERK2, through the use of PD98059 (a selective pharmacological inhibitor of ERK pathway), markedly abolished Nrf2 nuclear translocation induced by AS (Fig. 5). These results demonstrated that the ERK1/2 pathway is involved in AS-induced Nrf2/ARE pathway activation and in the protective effect against mild hyperoxia-induced endothelial damage although we cannot exclude the involvement of other mechanisms.

In conclusion, this study shows that anthocyanins and/or their metabolites, present in human serum after oral administration of a dietary plant-derived supplementation, can protect HUVECs against mild hyperoxia-induced 
alterations acting as cell signaling modulators and inducing activation of the Nrf2/ARE pathway. Thus, the present findings contribute to support the hypothesis that plant polyphenols, including anthocyanins, can play an important role in the prevention of diseases associated with oxidative stress, not due to their putative antioxidant properties but through activities and roles totally independent on such capacity and interacting with cell functions at different levels. A significant contribution is also given by the "bioreactor" model used to obtain these results, because it let to take into account the possible problems related to the drug oral availability and metabolism, as well as the possible synergisms between different components. Finally, the present results suggest the importance to plan dietary intervention based on plant-derived supplements which may efficiently prevent endothelial damage due to occupational or therapeutic exposure to mild hyperoxic conditions.

\section{References}

Altemeier WA, Sinclair SE (2007) Hyperoxia in the intensive care unit: why more is not always better. Curr Opin Crit Care 13:73-78

Canali R, Ambra R, Stelitano C, Mattivi F, Scaccini C, Virgili F (2007) A novel model to study the biological effects of red wine at the molecular level. British J Nutr 97:1053-1058

Canali R, Comitato R, Ambra R, Virgili F (2010) Red wine metabolites modulate NF-kappaB, activator protein-1 and cAMP response element-binding proteins in human endothelial cells. $\mathrm{Br}$ J Nutr 103:807-814

Cho HY, Jedlicka AE, Reddy SP, Kensler TW, Yamamoto M, Zhang LY, Kleeberger SR (2002) Role of NRF2 in protection against hyperoxic lung injury in mice. Am J Respir Cell Mol Biol $26: 175-182$

Dröge W (2002) Free radicals in the physiological control of cell function. Physiol Rev 82:47-95

Filomeni G, Piccirillo S, Rotilio G, Ciriolo MR (2012) p38(MAPK) and ERK1/2 dictate cell death/survival response to different prooxidant stimuli via p53 and $\mathrm{Nrf} 2$ in neuroblastoma cells $\mathrm{SH}-$ SY5Y. Biochem Pharmacol 83:1349-1357

Genestra M (2007) Oxyl radicals, redox-sensitive signalling cascades and antioxidants. Cell Signal 19:1807-1819

Gore A, Muralidhar M, Espey MG, Degenhardt K, Mantell LL (2010) Hyperoxia sensing: from molecular mechanisms to significance in disease. J Immunotoxicol 7:239-254

Limón-Pacheco JH, Hernández NA, Fanjul-Moles ML, Gonsebatt ME (2007) Glutathione depletion activates mitogen-activated protein kinase (MAPK) pathways that display organ-specific responses and brain protection in mice. Free Radic Biol Med 43:1335-1347

Livak KJ, Schmittgen TD (2001) Analysis of relative gene expression data using real-time quantitative PCR and the 2(-Delta Delta C(T)) Method. Methods 25:402-408
Morabito G, Trombetta D, Singh Brajendra K, Prasad Ashok K, Parmar Virinder S, Naccari C, Mancari F, Saija A, Cristani M, Firuzi O, Saso L (2010) Antioxidant properties of 4-methylcoumarins in in vitro cell-free systems. Biochimie 92:1101-1107

Oktyabrsky ON, Smirnova GV (2007) Redox regulation of cellular functions. Biochemistry 72:132-145

Pagano A, Barazzone-Argiroffo C (2003) Alveolar cell death in hyperoxia-induced lung injury. Ann NY Acad Sci 1010:405-416

Pendyala S, Moitra J, Kalari S, Kleeberger SR, Zhao Y, Reddy SP, Garcia JG, Natarajan V (2011) Nrf2 regulates hyperoxia-induced Nox4 expression in human lung endothelium: identification of functional antioxidant response elements on the Nox4 promoter. Free Radic Biol Med 50:1749-1759

Phillips PG, Tsan MF (1988) Hyperoxia causes increased albumin permeability of cultured endothelial monolayers. J Appl Physiol 64:1196-1202

Qin Y, Xia M, Ma J, Hao Y, Liu J, Mou H, Cao L, Ling W (2009) Anthocyanin supplementation improves serum LDL- and HDLcholesterol concentrations associated with the inhibition of cholesteryl ester transfer protein in dyslipidemic subjects. Am J Clin Nutr 90:485-492

Rahman I, Biswas SK, Kirkham PA (2006) Regulation of inflammation and redox signaling by dietary polyphenols. Biochem Pharmacol 72:1439-1452

Reddy NM, Kleeberger SR, Kensler TW, Yamamoto M, Hassoun PM, Reddy SP (2009) Disruption of Nrf2 impairs the resolution of hyperoxia-induced acute lung injury and inflammation in mice. J Immunol 182:7264-7271

Shen G, Hebbar V, Nair S, Xu C, Li W, Lin W, Keum YS, Han J, Gallo MA, Kong AN (2004) Regulation of Nrf2 transactivation domain activity. The differential effects of mitogen-activated protein kinase cascades and synergistic stimulatory effect of Raf and CREB-binding protein. J Biol Chem 279:23052-23060

Speciale A, Canali R, Chirafisi J, Saija A, Virgili F, Cimino F (2010) Cyanidin-3-O-glucoside protection against TNF- $\alpha$-induced endothelial dysfunction: involvement of nuclear factor- $\kappa \mathrm{B}$ signaling. J Agric Food Chem 58:12048-12054

Speciale A, Anwar S, Ricciardi E, Chirafisi J, Saija A, Cimino F (2011a) Cellular adaptive response to glutathione depletion modulates endothelial dysfunction triggered by TNF- $\alpha$. Toxicol Lett 207:291-297

Speciale A, Chirafisi J, Saija A, Cimino F (2011b) Nutritional antioxidants and adaptive cell responses: an update. Curr Mol Med 11:770-789

Surh YJ, Kundu JK, Na HK (2008) Nrf2 as a master redox switch in turning on the cellular signaling involved in the induction of cytoprotective genes by some chemopreventive phytochemicals. Planta Med 74:1526-1539

Thom SR (2009) Oxidative stress is fundamental to hyperbaric oxygen therapy. J Appl Physiol 106:988-995

Tomaino A, Martorana M, Arcoraci T, Monteleone D, Giovinazzo C, Saija A (2010) Antioxidant activity and phenolic profile of pistachio (Pistacia vera L., variety Bronte) seeds and skins. Biochimie 92:1115-1122

Wells PG, McCallum GP, Chen CS, Henderson JT, Lee CJ, Perstin J, Preston TJ, Wiley MJ, Wong AW (2009) Oxidative stress in developmental origins of disease: teratogenesis, neurodevelopmental deficits, and cancer. Toxicol Sci 108:4-18 